\title{
dspace.vutbr.cz
}

\section{Verification of Nine-phase PMSM Model in d-q Coordinates with Mutual Couplings}

\author{
KOZOVSKÝ, M.; BLAHA, P.; VÁCLAVEK, P
}

6th IEEE International Conference on Control System, Computing and Engineering (ICCSCE 2016) pp. 78-83

ISBN: 978-1-5090-1177-3

DOl: http://dx.doi.org/10.1109/ICCSCE.2016.7893548

Accepted manuscript

(C)2016 IEEE. Personal use of this material is permitted. Permission from IEEE must be obtained for all other uses, in any current or future media, including reprinting/republishing this material for advertising or promotional purposes, creating new collective works, for resale or redistribution to servers or lists, or reuse of any copyrighted component of this work in other works. Matús Kozovský, Petr Blaha, Pavel Václavek, " Verification of Nine-phase PMSM Model in d-q Coordinates with Mutual Couplings", 6th IEEE International Conference on Control System, Computing and Engineering (ICCSCE 2016), pp. 78-83, 2016. DOI: 10.1109/ICCSCE.2016.7893548. Final version is available at http://ieeexplore.ieee.org/document/7893548/ 


\title{
Verification of Nine-phase PMSM Model in $d-q$ Coordinates with Mutual Couplings
}

\author{
Matus Kozovsky, Petr Blaha and Pavel Vaclavek \\ CEITEC BUT \\ Brno University of Technology \\ Purkynova 123, Brno 612 00, Czech Republic \\ Phone: +420 541146454 \\ Emails: matus.kozovsky@ceitec.vutbr.cz, petr.blaha@ceitec.vutbr.cz, pavel.vaclavek@ceitec.vutbr.cz \\ URL: http://www.ceitec.eu/
}

\begin{abstract}
Electric motors with more than three phases have many advantages comparing to ordinary three-phase motor. For this reason it is natural to pay attention to them and to work on advanced control methods. Control algorithms development requires to operate with the model of motor. This paper presents the modeling concept of the nine-phase permanent magnet synchronous motor (PMSM) in three times three-phase arrangement fed by nine-phase voltage source inverter (VSI). Magnetic interaction between phases and phase groups had to be taken into account. The paper also provides relationship between model parameters and real motor ones, depending on the windings configuration. It shows the comparison between the simulation and experimental results with real motor. Used method of motor parameters measurement and the subsequent conversion of these parameters into $d-q$ coordinates is clarified in this paper.
\end{abstract}

Index Terms-Modeling, Multiphase, d-q model, 3x3-phase PMSM,

\section{INTRODUCTION}

Multi-phase machines are now commonly used for high power drive applications as turbo-compressors and ultra high speed elevators because of their high efficiency [1]. Control of these motors can also be adjusted to operate during fault conditions. They can be also used in the automotive industry due to their reliability, efficiency and redundancy. Multi-phase motors can operate at a lower supply voltage or lower supply currents than standard three-phase motors with equal power [2]. To understand its functionality, it is necessary to know the motor model and its behavior. To create a fault-tolerant control algorithm it is necessary to know what currents and voltages motor can generate [3]. Motor which will be discussed here is permanent magnet synchronous motor (PMSM). With this type of motor, a model in $d-q$ coordinates is usually created using Park transformation. This coordinate system is connected to the rotor and allows control to a constant values of currents [4], [5], [6]. The PMSM is also designed so as to suppress the effect of nonlinearities. Finite element (FE) method can be used to create the model which is computationally demanding

The research was supported by ECSEL JU under the project 662192 3Ccar Integrated Components for Complexity Control in affordable electrified cars and by FEKT/FSI-J-16-3694.

The research results were verified in simulation using AVL CRUISE M simulation SW provided by AVL within University Partnership Program.
[7]. The second possible way of creating the model is using differential equations [8] and analytical approach [9]. The second approach is needed for control algorithms development and it will be treated in details in this paper.

\section{A. Multi-phase motor vs multiple k-phase motor}

It is important to recognize the difference between multiphase electric motor and motor with multiple $k$-phase winding. When we speak about classical multi-phase motor we usually expect electrical star connection of all windings. Windings in this type of motor are uniformly distributed in the stator. Fig. 1(a) shows the phase shift between windings for balanced fivephase motor as an example. The angle depends on total number of phases. While focusing on reliability, this arrangement has the disadvantage since potential fault can affect the winding constantly connected to DC-bus power or ground. This affects currents in other windings and it also changes middle junction voltage [8], [10].

On the other hand, multiple $k$-phase motor has the individual coil systems (usually three-phase) electrically separated and there is mutual induction coupling between subsystems. One subsystem can be completely electrically disconnected from power source in case of fault. Therefore, subsystem is not energized and do not generate magnetic field. The motor continues to operate with lower power [10].

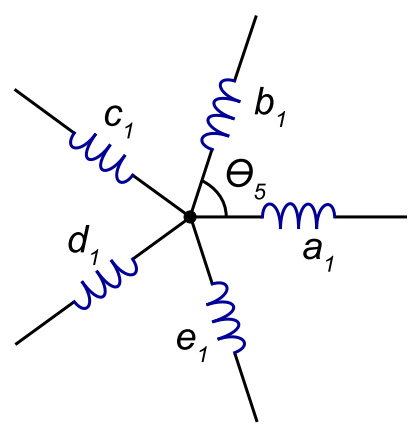

(a)

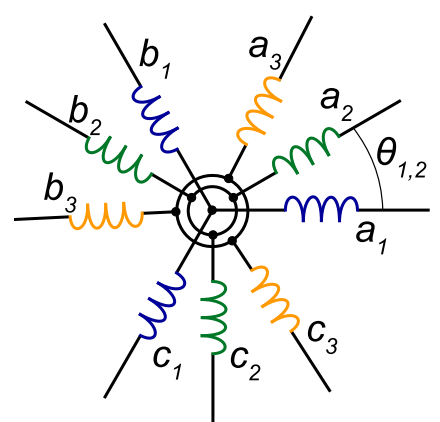

(b)
Fig. 1: Five-phase winding and triple three-phase winding. 


\section{B. Mechanical Construction}

The multiple $k$-phase motors may have different arrangements of windings in the stator. In this article we will deal with triple three-phase motors. Obviously the mutual angle between the windings can be given in mechanical degrees or in electrical degrees. Electrical angle and mechanical angle are mostly different. Mechanical displacement depends on the number of pole-pairs. The model is created in $d$ - $q$ coordinates and all variables are transformed from $a b c$ space to $d-q$ coordinates [11], [12].

\section{ANALYSIS OF TRIPLE 3-PHASE WindingS}

Fig. 1 (b) shows possible windings alignment of triple 3phase stator. Final model and equations are in $d-q$ coordinates. The $q$-axis leads the $d$-axis by $\pi / 2$ electrical radians and actual angle to stator coordinates depends on rotor position. While we apply $d-q$ transformation on base $a b c$ space we get the equation (1). After the transformation of the threephase system to a $d-q$ coordinates we obtain two equations for each subsystem. From the three subsystems of three-phase motors, six equations are received after the transformation. Three equations for $d$-axis and three for $q$-axis. The $j$-th subsystem can be described as

$$
\begin{aligned}
& v_{d_{j}}=R_{j} i_{d_{j}}+\frac{d \psi_{d j}}{d t}-\omega \psi_{q_{j}} \\
& v_{q_{j}}=R_{j} i_{q_{j}}+\frac{d \psi_{q j}}{d t}+\omega \psi_{d_{j}}
\end{aligned}
$$

where

$$
j=\{1,2,3\}
$$

Variable $v$ represents voltages id $d$ and $q$ coordinates, $R$ is winding resistance, $i$ represents electric currents and $\psi$ is magnetic flux, $\omega$ is electrical speed of rotation.

The same equations are used for other subsystems but with different indexes $j$. When transforming ordinary three-phase motor, there is just one $d$-winding and one $q$-winding and the flux-linkages are given by following equations

$$
\begin{aligned}
& \psi_{d 1}=\Psi_{M d 1}+L_{d 1} i_{d 1} \\
& \psi_{q 1}=L_{q 1} i_{q 1}
\end{aligned}
$$

$\Psi_{M}$ represents back electromotive force constant, $\mathrm{L}$ is inductance of windings.

When transforming multiple 3-phase systems it is necessary take into consideration mutual inductances. It leads to extension of equations (2). For triple subsystem we can write the

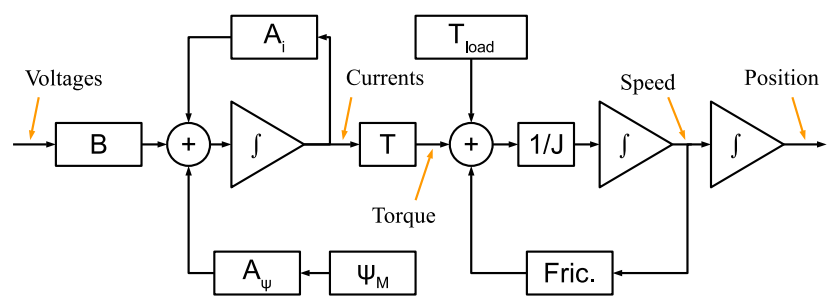

Fig. 2: Block diagram of motor model. following equation. $\mathrm{M}$ represents mutual inductance between subsystems windings.

$$
\begin{aligned}
& \psi_{d 1}=\Psi_{M d 1}+L_{d 1} i_{d 1}+M_{d 1 d 2} i_{d 2}+M_{d 1 d 3} i_{d 3} \\
& \psi_{q 1}=L_{q 1} i_{q 1}+M_{q 1 q 2} i_{q 2}+M_{q 1 q 3} i_{q 3}
\end{aligned}
$$

For simplification it will be considered that all subsystems have the same electrical characteristic and that mutual inductances are the same. This assumption must comply with the real motor. After adjusting the flux equations (3) and their substitution to the voltage equation (1) we obtain equations (4a-4f). They show the relationships between individual voltages and currents.

$$
\begin{aligned}
v_{d 1}= & L_{d} \frac{d i_{d 1}}{d t}+M_{d} \frac{d i_{d 2}}{d t}+M_{d} \frac{d i_{d 3}}{d t}+R i_{d 1}- \\
& \omega\left(L_{q} i_{q 1}+M_{q} i_{q 2}+M_{q} i_{q 3}\right) \\
v_{d 2}= & M_{d} \frac{d i_{d 1}}{d t}+L_{d} \frac{d i_{d 2}}{d t}+M_{d} \frac{d i_{d 3}}{d t}+R i_{d 1}- \\
& \omega\left(M_{q} i_{q 1}+L_{q} i_{q 2}+M_{q} i_{q 3}\right) \\
v_{d 3}= & M_{d} \frac{d i_{d 1}}{d t}+M_{d} \frac{d i_{d 2}}{d t}+L_{d} \frac{d i_{d 3}}{d t}+R i_{d 1}- \\
& \omega\left(M_{q} i_{q 1}+M_{q} i_{q 2}+L_{q} i_{q 3}\right) \\
v_{q 1}= & \omega\left(L_{d} i_{d 1}+M_{d} i_{d 2}+M_{d} i_{d 3}+\Psi_{M}\right)+ \\
& L_{q} \frac{d i_{q 1}}{d t}+M_{q} \frac{d i_{q 2}}{d t}+M_{q} \frac{d i_{q 3}}{d t}+R i_{q 1} \\
v_{q 2}= & \omega\left(M_{d} i_{d 1}+L_{d} i_{d 2}+M_{d} i_{d 3}+\Psi_{M}\right)+ \\
& M_{q} \frac{d i_{q 1}}{d t}+L_{q} \frac{d i_{q 2}}{d t}+M_{q} \frac{d i_{q 3}}{d t}+R i_{q 1} \\
v_{q 3}= & \omega\left(M_{d} i_{d 1}+M_{d} i_{d 2}+L_{d} i_{d 3}+\Psi_{M}\right)+ \\
& M_{q} \frac{d i_{q 1}}{d t}+M_{q} \frac{d i_{q 2}}{d t}+L_{q} \frac{d i_{q 3}}{d t}+R i_{q 1}
\end{aligned}
$$

Given equations (4) are not well suited for modelling and numerical simulations. Therefore, it is necessary to convert them to state space description form. Currents in $d$ and $q$ coordinates can be selected as state variables to form state vector $\boldsymbol{i}=\left(i_{d 1}, i_{q 1}, i_{d 2}, i_{q 2}, i_{d 3}, i_{q 3}\right)^{T}$. Their time derivatives depend on the actual currents, input voltages, rotor flux and the actual speed of the motor. The block diagram of nonlinear state space model is shown in Fig. 2. Full Matlab schematic can be wired according to the model using all necessary matrices.

The matrix $\boldsymbol{B}$ relates the currents time derivatives with input voltages $\boldsymbol{v}=\left(v_{d 1}, v_{q 1}, v_{d 2}, v_{q 2}, v_{d 3}, v_{q 3}\right)^{T}$. It is called system input matrix. This matrix also shows an input mutual linkage between subsystems. 


$$
\boldsymbol{B}=\left[\begin{array}{cccccc}
\sigma_{4} & 0 & \sigma_{2} & 0 & \sigma_{2} & 0 \\
0 & \sigma_{3} & 0 & \sigma_{1} & 0 & \sigma_{1} \\
\sigma_{2} & 0 & \sigma_{4} & 0 & \sigma_{2} & 0 \\
0 & \sigma_{1} & 0 & \sigma_{3} & 0 & \sigma_{1} \\
\sigma_{2} & 0 & \sigma_{2} & 0 & \sigma_{4} & 0 \\
0 & \sigma_{1} & 0 & \sigma_{1} & 0 & \sigma_{3}
\end{array}\right]
$$

where

$$
\begin{aligned}
& \sigma_{1}=-\frac{M_{q}}{L_{q}^{2}+L_{q} M_{q}-2 M_{q}^{2}} \\
& \sigma_{2}=-\frac{M_{d}}{L_{d}^{2}+L_{d} M_{d}-2 M_{d}^{2}} \\
& \sigma_{3}=-\frac{L_{q}+M_{q}}{L_{q}^{2}+L_{q} M_{q}-2 M_{q}^{2}} \\
& \sigma_{4}=\frac{L_{d}+M_{d}}{L_{d}^{2}+L_{d} M_{d}-2 M_{d}^{2}}
\end{aligned}
$$

Matrix $\boldsymbol{B}$ shows that $d$-axis voltages influence only $d$-axis currents. This also holds for $q$-axis quantities. The interaction between $d$ and $q$ axis is ideally zero because mutual inductance between the coils of these axes should be zero. Mutual inductances between the subsystems are reflected by matrix $\boldsymbol{A}_{i}$. This matrix is a feedback matrix of links between individual currents and its time derivatives and it has following form

$$
\boldsymbol{A}_{i}\left(\omega_{e}\right)=\left[\begin{array}{llllll}
\sigma_{8} & \sigma_{6} & \sigma_{4} & \sigma_{2} & \sigma_{4} & \sigma_{2} \\
\sigma_{5} & \sigma_{7} & \sigma_{1} & \sigma_{3} & \sigma_{1} & \sigma_{3} \\
\sigma_{4} & \sigma_{2} & \sigma_{8} & \sigma_{6} & \sigma_{4} & \sigma_{2} \\
\sigma_{1} & \sigma_{3} & \sigma_{5} & \sigma_{7} & \sigma_{1} & \sigma_{3} \\
\sigma_{4} & \sigma_{2} & \sigma_{4} & \sigma_{2} & \sigma_{8} & \sigma_{6} \\
\sigma_{1} & \sigma_{3} & \sigma_{1} & \sigma_{3} & \sigma_{5} & \sigma_{7}
\end{array}\right]
$$

where

$$
\begin{aligned}
& \sigma_{1}=\frac{\omega_{e}\left(L_{d} M_{q}-L_{q} M_{d}\right)}{L_{q}^{2}+L_{q} M_{q}-2 M_{q}^{2}} \\
& \sigma_{2}=\frac{\omega_{e}\left(L_{d} M_{q}-L_{q} M_{d}\right)}{L_{d}^{2}+L_{d} M_{d}-2 M_{d}^{2}} \\
& \sigma_{3}=\quad \frac{M_{q} R}{L_{q}^{2}+L_{q} M_{q}-2 M_{q}^{2}} \\
& \sigma_{4}=\quad \frac{M_{d} R}{L_{d}^{2}+L_{d} M_{d}-2 M_{d}^{2}} \\
& \sigma_{5}=-\frac{\omega_{e}\left(L_{d} L_{q}+L_{d} M_{q}-2 M_{d} M_{q}\right)}{L_{q}^{2}+L_{q} M_{q}-2 M_{q}^{2}} \\
& \sigma_{6}=\frac{\omega_{e}\left(L_{q} L_{d}+L_{q} M_{d}-2 M_{d} M_{q}\right)}{L_{d}^{2}+L_{d} M_{d}-2 M_{d}^{2}} \\
& \sigma_{7}=\quad-\frac{L_{q} R+M_{q} R}{L_{q}^{2}+L_{q} M_{q}-2 M_{q}^{2}} \\
& \sigma_{8}=\quad-\frac{L_{d} R+M_{d} R}{L_{d}^{2}+L_{d} M_{d}-2 M_{d}^{2}}
\end{aligned}
$$

This feedback state matrix contains nonlinear components in presence of electrical speed $\omega_{e}$ since it will be later on selected as another state variable forming mechanical part of the motor model.

The matrix $\boldsymbol{A}_{\Psi}$ reflects influence of back electromotive force (back-EMF). It is the voltage, or electromotive force, that pushes against the magnetic field which induces it. In this case it is due to the rotating magnetic field generated by the rotor. This voltage also indicates the maximum speed that motor can rotate. When back-EMF voltage reaches critical level, the DC-bus voltage is applied to balance back-EMF and no voltage remains to generate torque. This part is shown in Fig. 2 as matrix $\boldsymbol{A}_{\Psi}$ with motor voltage constant $\Psi_{M}$ and its elements are as follows

$$
\boldsymbol{A}_{\Psi}=\left[\begin{array}{llllll}
0 & \sigma & 0 & \sigma & 0 & \sigma
\end{array}\right]^{T}
$$

where

$$
\sigma=\frac{\omega_{e}\left(L_{q}-M_{q}\right)}{L_{q}^{2}+L_{q} M_{q}-2 M_{q}^{2}}
$$

The behavior of electrical part of the motor can be described using nonlinear state equation

$$
\frac{d \boldsymbol{i}}{d t}=\boldsymbol{A}_{i}\left(\omega_{e}\right) \boldsymbol{i}+\boldsymbol{B} \boldsymbol{v}+\boldsymbol{A}_{\Psi} \Psi_{M}
$$

The mechanical part of the model requires conversion from motor currents to motor torque. The torque is a vector product of the magnetic flux of the motor (joint contribution of permanent magnet and currents) and the currents. This relationship for single 3-phase PMSM is given by the following equation

$$
T=\frac{3}{2} p\left(\Psi_{d} i_{q}-\Psi_{q} i_{d}\right)
$$

Torque calculation of triplex 3-phase motor is more complicated. Torque contribution must be considered in every subsystem. The resulting formula for calculating the torque produced by the motor is given as

$$
\begin{aligned}
T_{m o t}= & \frac{3}{2} p\left(\Psi_{M}\left(i_{q 1}+i_{q 2}+i_{q 3}\right)+\right. \\
& \left(i_{d 1} i_{q 1}+i_{d 2} i_{q 2}+i_{d 3} i_{q 3}\right)\left(L_{d}-L_{q}\right)+ \\
& \left(i_{d 1} i_{q 2}+i_{d 2} i_{q 1}+i_{d 1} i_{q 3}+\right. \\
& \left.\left.i_{d 3} i_{q 1}+i_{d 2} i_{q 3}+i_{d 3} i_{q 2}\right)\left(M_{d}-M_{q}\right)\right)
\end{aligned}
$$

The angular acceleration $\varepsilon_{e}$ of the rotor is time derivative of electrical speed $\omega_{e}$ and it depends on moment of inertia $J$ and on resulting torque connected to the rotor. The resulting torque is given as a sum of the motor torque $T_{m o t}$, the load torque $T_{\text {load }}$ and the friction torque $T_{\text {fric }}$

$$
\varepsilon_{e}=\frac{d \omega_{e}}{d t}=\frac{d^{2} \varphi_{e}}{d t^{2}}=\frac{1}{J}\left(T_{m o t}+T_{\text {load }}+T_{\text {fric }}\right)
$$

As it can be seen from Fig. 2, the friction torque is assumed to be proportional to the speed but it has opposite direction. The equation (11) represents the mechanical part of the motor model (having electrical rotor position $\varphi_{e}$ and speed $\omega_{e}$ as state variables) and together with equation (8) they form complete non-linear electro-mechanical motor model with eight state variables. 


\section{Model Parameters Measurement And COMPUTATION}

The model described in previous section is established in $d$ $q$ coordinates. If we want to simulate the real motor behavior we need to specify its parameters. The measurement on the motor can give us only parameters in $a b c$ coordinates. These parameters need to be re-calculated to $d-q$ coordinates and then they can be used in derived model.

The re-calculation is realized using the transformation matrix $\boldsymbol{T}$ [10]. This matrix depends on mutual shift of individual $k$-phase windings $\eta$ and on mutual shift between $k$-phase subsystems $\theta$. Transformation matrix has the following form

$$
\boldsymbol{T}=\frac{2}{3}\left[\begin{array}{ccc}
\cos (\theta) & \cos (\theta-\eta) & \cos (\theta+\eta) \\
-\sin (\theta) & -\sin (\theta-\eta) & -\sin (\theta+\eta) \\
1 / 2 & 1 / 2 & 1 / 2
\end{array}\right]
$$

where

$$
\eta=120^{\circ}
$$

for 3-phase subsystems with $120^{\circ}$ angle between the windings.

Typically, $a b c$ to $d$ - $q$ transformation is used for three-phase motor linkages $\boldsymbol{\Psi}_{a b c}$ and curents $\boldsymbol{i}_{a b c}$ and it can be expressed as follows

$$
\begin{gathered}
\boldsymbol{\Psi}_{a b c}=\boldsymbol{L}_{a b c} \boldsymbol{i}_{a b c} \\
\boldsymbol{i}_{d q 0}=\boldsymbol{T} \boldsymbol{i}_{a b c} ; \quad \boldsymbol{i}_{a b c}=\boldsymbol{T}^{-1} \boldsymbol{i}_{d q 0} \\
\boldsymbol{\Psi}_{d q 0}=\boldsymbol{T} \boldsymbol{\Psi}_{a b c}= \\
\boldsymbol{L}_{d q 0}=\boldsymbol{T} \boldsymbol{L}_{a b c} \boldsymbol{T}^{-1} \boldsymbol{i}_{d q 0}
\end{gathered}
$$

Equation (13d) shows how to use the matrix transformation for one subsystem. Three transformation matrixes are needed to recompute parameters of triple 3-phase subsystems.

$$
\boldsymbol{L}=\left[\begin{array}{ccc}
\boldsymbol{T}_{1} \boldsymbol{L}_{1} \boldsymbol{T}_{1}^{-1} & \boldsymbol{T}_{1} \boldsymbol{M}_{12} \boldsymbol{T}_{2}^{-1} & \boldsymbol{T}_{1} \boldsymbol{M}_{13} \boldsymbol{T}_{3}^{-1} \\
\boldsymbol{T}_{2} \boldsymbol{M}_{21} \boldsymbol{T}_{1}^{-1} & \boldsymbol{T}_{2} \boldsymbol{L}_{2} \boldsymbol{T}_{2}^{-1} & \boldsymbol{T}_{2} \boldsymbol{M}_{23} \boldsymbol{T}_{3}^{-1} \\
\boldsymbol{T}_{3} \boldsymbol{M}_{31} \boldsymbol{T}_{1}^{-1} & \boldsymbol{T}_{3} \boldsymbol{M}_{32} \boldsymbol{T}_{2}^{-1} & \boldsymbol{T}_{3} \boldsymbol{L}_{3} \boldsymbol{T}_{3}^{-1}
\end{array}\right]
$$

In this equation, $\boldsymbol{L}_{1}$ to $\boldsymbol{L}_{3}$ represent the inductance matrices of the windings in the subsystem. Mutual inductance matrix between subsystems $i$ and $j$ is expressed by matrix $\boldsymbol{M}_{i j}$. Transformation matrices $\boldsymbol{T}_{1}$ to $\boldsymbol{T}_{3}$ are distinguished by the mutual shift between $k$-phase subsystems $\theta$.

\section{MODEL VERIFICATION}

Several tests were performed with a real motor to verify the model behavior. The tested motor was TGT3-0130-1536/T4X1-ES28 from TG Drives company. This motor is normally three-phase but the windings were during the production disconnected and taken out from the motor. It enables to connect the motor in differen configurations and also to simulate different faults. The same conditions were settled for the model in simulations for objective comparison. A field oriented control algorithm was used in several cases. Tests were carried out to verify the friction dynamic properties, windings electric properties and rotor magnetic properties.

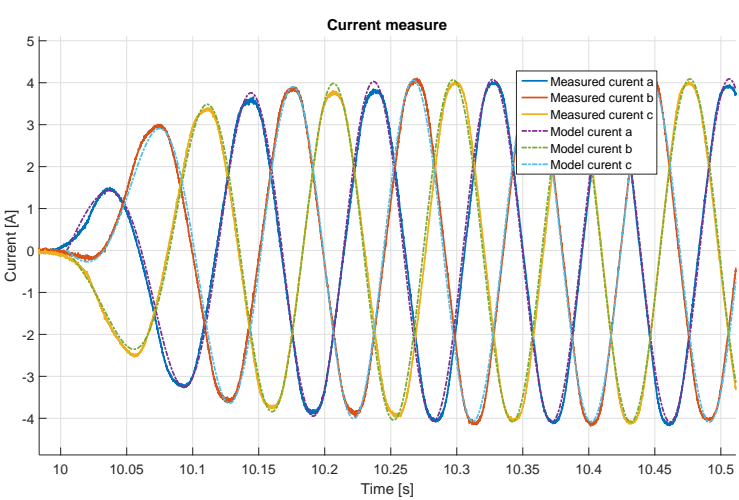

Fig. 3: Motor currents in one 3-phase subsystem when starting with external dynamometer (torque $0.5 \mathrm{Nm}$ )

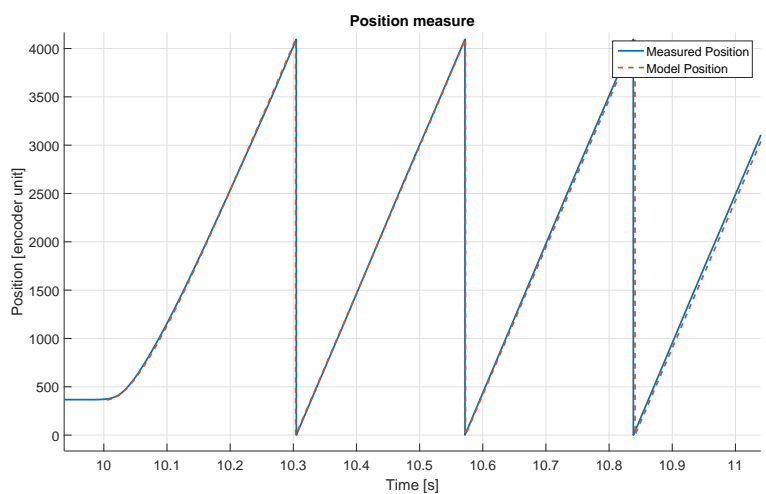

Fig. 4: Motor position when starting external dynamometer (torque $0.5 \mathrm{Nm}$ )

A $2 \mathrm{~kW}$ dynamometer DYNOFIT ASD 2000-2 from VUES company was used to create test conditions.

\section{A. External Torque Applied to Short Circuited Motor}

In this test, the motor was loaded with a constant torque produced by the dynamometer. The motor had all phases connected to the ground (negative pole of the DC-bus) by the inverter. The motor acts as a brake in this configuration and its breaking torque depends on the actual speed. The torque produced by the dynamometer was set from 0 to $0.5 \mathrm{Nm}$.

After starting a dynamometer, the motor began to generate currents caused by the back-EMF. Phase currents, the rotor position and its rotation speed were measured. The measurement results were compared with the simulation results. Fig. 3 shows comparison of currents generated by back-EMF. It can be seen that simulated currents correspond to the measured ones. Fig. 4 shows the position of the rotor compared to the simulated results. In this test it was also verified that the braking torque generated by the motor is consistent with the simulations results which implies equal resulting speeds. Speed comparison can be seen in Fig. 5. Measured speed slightly ripples due to unbalanced torque distribution per rotor revolution since period of ripples corresponds to one mechanical turn. This test verifies the model dynamic characteristics and shows very good fit with real motor behavior. 


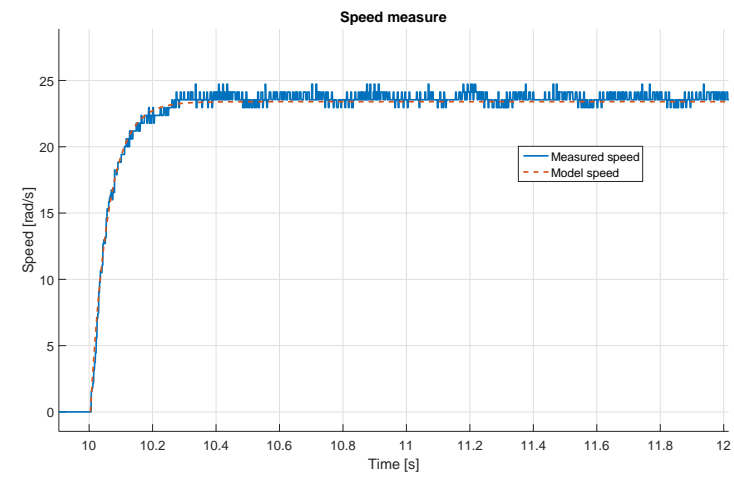

Fig. 5: Motor speed when starting external dynamometer (torque $0.5 \mathrm{Nm}$ )

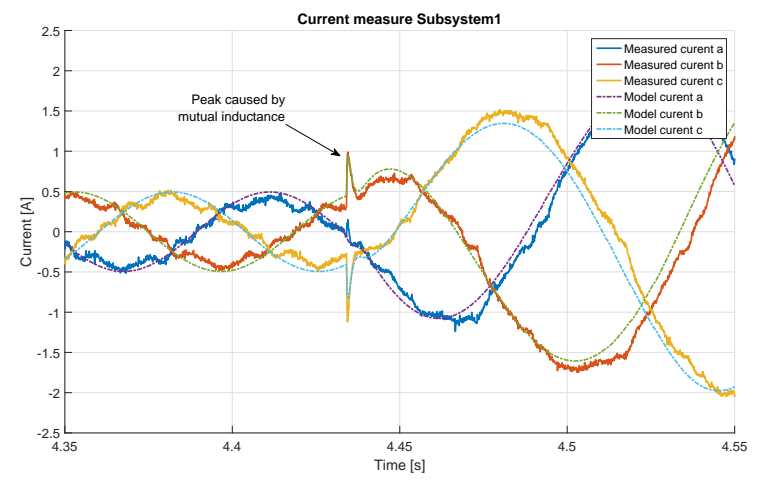

Fig. 6: Motor currents in first subsystem when short circuiting the third subsystem.

\section{B. Short Circuiting of One Subsystem}

In the second test, the motor was running at the required speed of $24 \mathrm{rad} / \mathrm{s}$. Speed setpoint was assured with PI speed controller. The same controller was used to control the motor model in simulations. The output from speed controller (required current) was limited to $2 \mathrm{~A}$ in each subsystem in order to provide the test (the maximum current of all subsystems is $6 \mathrm{~A}$ for selected motor). During such operation, the third subsystems was short-circuited as in previous test which caused its internal breaking torque generation and its fast increase of currents produced by the back-EMF. These changes in currents were reflected in other subsystems due to mutual inductances. Breaking torque implies that the currents of the other two motor subsystems must increase in order to supply the necessary torque which short-circuited subsystem draws. This is demonstrated in Fig. 6. The short circuit was forced around the time of $4.43 \mathrm{~s}$ where measured and also simulated peaks in currents can be observed. Fig. 7 shows fast increase in the currents of short circuited subsystem.

The motor is working without load torque until short circuiting third subsystem. Distortion of currents on a real motor is visible due to uncompensated dead-time. Final motor speed is reduced by short-circuited subsystem because the remaining two sub-systems are unable to supply sufficient torque. Their maximum torque is limited by the maximum current set on

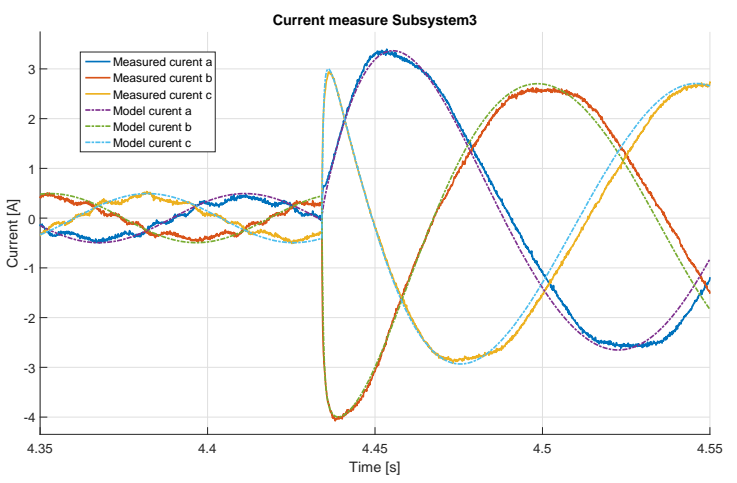

Fig. 7: Currents in the third (short circuited) subsystem

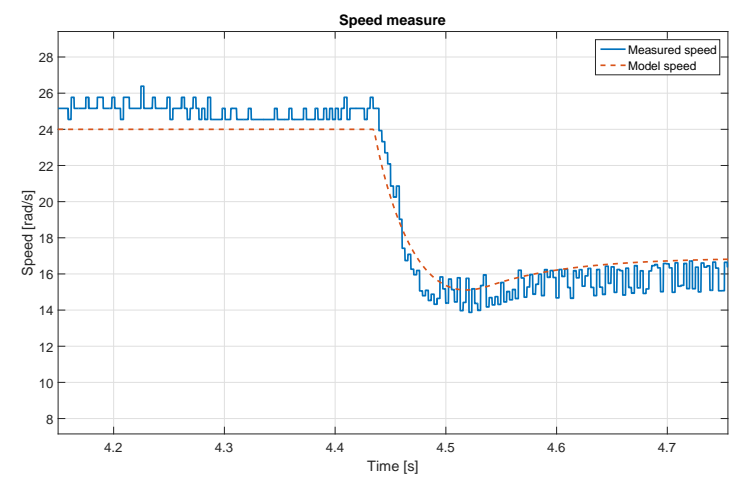

Fig. 8: Speed response under short circuited operation of one subsystem

the controller. The resulting speed is then stabilized around 16 $\mathrm{rad} / \mathrm{s}$. This situation can be seen in Fig. 8. This test showed that simulated mutual inductances between individual subsystems fit well to the real motor behavior.

\section{Applying Load Torque Changes}

The last test which was carried out shows the behavior of the model and the real motor with activated current and speed controllers in case of load torque changes. The required speed of the motor was set to $24 \mathrm{rad} / \mathrm{s}$. After reaching a constant speed, a load torque on the dynamometer was set from 0 to $0.15 \mathrm{Nm}$ (time $12.07 \mathrm{~s}$ ). A short time drop of the motor speed occurred whilst increasing a load torque. This drop depends on the electrical and mechanical characteristics of the motor and also depends on the speed and current controller settings. Whereas the used controller is identical with the inverter implemented controller therefore their characteristics can be compared. The increase in torque causes a decrease in the speed to approximately $16 \mathrm{rad} / \mathrm{s}$ which can be seen in Fig. 11. The measured and simulated results of the speed are the same which proves the affectivity of the model. We can also see in this figure that the real motor speed contains ripples. The period of these ripples is about $0.27 \mathrm{~s}$. It corresponds to a rotation speed of approximately $23.3 \mathrm{rad} / \mathrm{s}$. Since the motor operates at $24 \mathrm{rad} / \mathrm{s}$ it can be deduced that the ripples are caused by unbalanced torque during one revolution of the rotor and low value of inertia achieved on a test bench. Same 


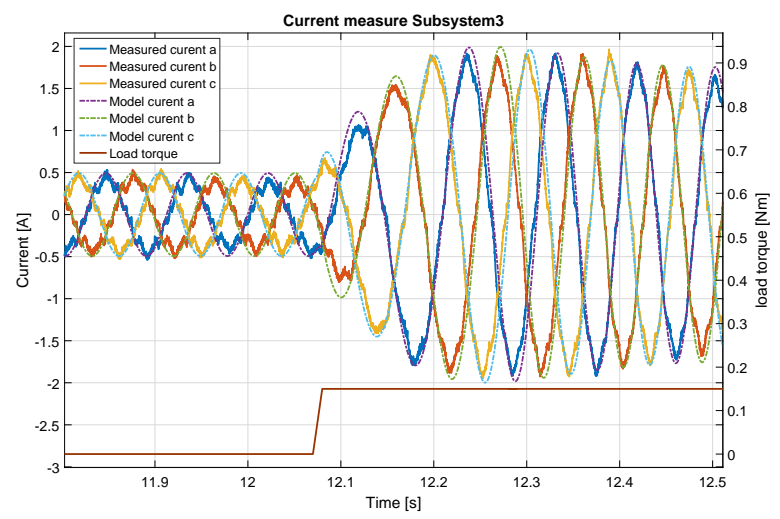

Fig. 9: Motor currents at $0.15 \mathrm{Nm}$ applied load torque

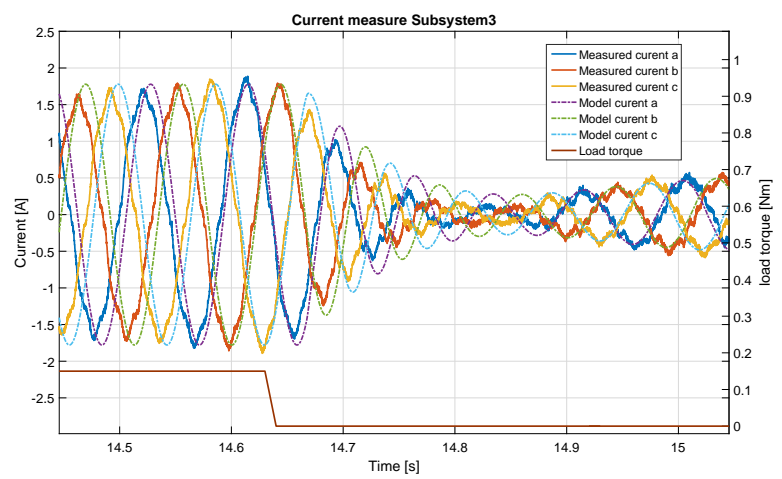

Fig. 10: Motor currents at no-load torque applied in one subsystem

ripples were observed in the first test. The load torque step change back to $0 \mathrm{Nm}$ is applied at time $14.63 \mathrm{~s}$. The short speed change in the opposite direction can be observed. Fig. 9 and 10 show the motor currents when both load torque step changes were applied.

\section{CONCLUSION}

A mathematical model of triple 3-phase motor was derived, outlined and verified in this paper. An individual state space description matrices values were calculated with the help of MuPad which is a part of Symbolic Toolbox in MATLAB. Input matrix and feedback matrix were derived. The motor torque was evaluated for triple 3-phase PMSM. The model parameters were adjusted according to the real motor. The model parameters were set according to the measured and transformed motor parameters. The mathematical model was created in MATLAB/SIMULINK based on derived equations. The developed model was also realized as MATLAB Level-2 $\mathrm{S}$-function but it did not increase the computational speed of simulation experiments.

Three experimental tests were carried out to verify real motor compliance with the mathematical model. The tests proved inaccuracies of a model at low motor load. These problems are caused mainly by uncompensated dead-time. Tests also showed that the modeled mutual inductances are identified

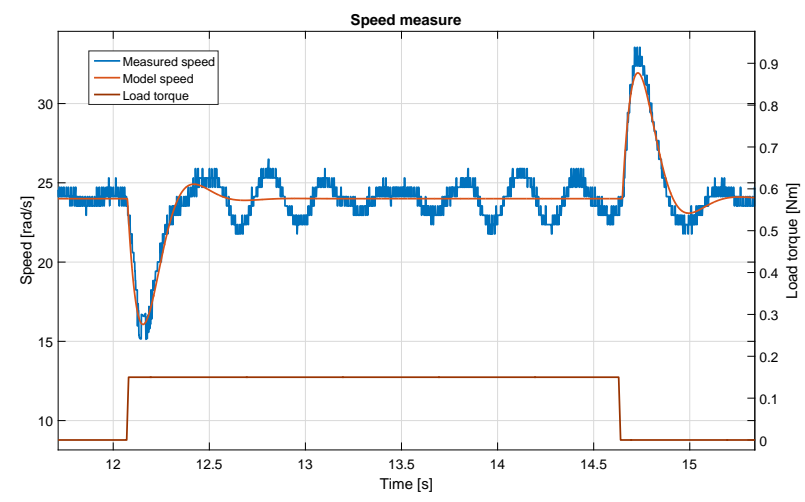

Fig. 11: Speed reaction to torque changes

properly due to good correspondence between simulated and measured currents.

The future work will use derived model to realize decoupling for nine phase motor. This application can detect imperfections in the model. Also it may prove that the model needs to include other physical effects. Realised model needs to be adjusted in that case. In summary the knowledge of the model can be used in the design of advanced control.

\section{REFERENCES}

[1] E. Jung, H. Yoo, S.-K. Sul, H.-S. Choi, and Y.-Y. Choi, A Nine-Phase Permanent-Magnet Motor Drive System for an Ultrahigh-Speed Elevator, IEEE Transactions on Industry Applications, vol. 48, no. 3, pp. 987995 , May 2012.

[2] R. R. Bastos, R. M. Valle, S. L. Nau, and B. J. C. Filho, Modelling and analysis of a nine-phase induction motor with third harmonic current injection, in 2015 9th International Conference on Power Electronics and ECCE Asia (ICPE-ECCE Asia), 2015, pp. 688694.

[3] F. Yu, M. Cheng, F. Li, K. T. Chau, J. Huang, and W. Hua, Fault tolerant control of harmonic injected nine-phase flux switching permanent magnet motor drive system, in 2014 17th International Conference on Electrical Machines and Systems (ICEMS), 2014, pp. 31173122.

[4] J. Liu and W. Chen, Generalized DQ model of the permanent magnet synchronous motor based on extended park transformation, in 2013 1st International Future Energy Electronics Conference (IFEEC), 2013, pp. 885890.

[5] Tessarolo, A., Bortolozzi, M., Contin, A. (2013). Modeling of split-phase machines in Parks coordinates. Part I: Theoretical foundations. Eurocon 2013 (pp. 13081313). IEEE. doi:10.1109/EUROCON.2013.6625148

[6] Tessarolo, A., Bortolozzi, M., Contin, A. (2013). Modeling of split-phase machines in Parks coordinates. Part II: Equivalent circuit representation. Eurocon 2013 (pp. 13141319). IEEE. doi:10.1109/EUROCON.2013.6625149

[7] M. Fasil, C. Antaloae, N. Mijatovic, B. B. Jensen, and J. Holboll, Improved dq-Axes Model of PMSM Considering Airgap Flux Harmonics and Saturation, IEEE Transactions on Applied Superconductivity, pp. 11, 2016.

[8] Tessarolo, A., Bortolozzi, M., Contin, A. (2013). Modeling of split-phase machines in Parks coordinates. Part I: Theoretical foundations. Eurocon 2013 (pp. 13081313). IEEE. doi:10.1109/EUROCON.2013.6625148

[9] Kim, H., Shin, K., Englebretson, S., Frank, N., Arshad, W. (2013). Analytical model of multiphase permanent magnet synchronous machines for energy and transportation applications. 2013 International Electric Machines Drives Conference (pp. 172179). IEEE. doi:10.1109/IEMDC.2013.6556250

[10] T. J. E. Miller and M. I. McGilp, Analysis of multi-phase permanentmagnet synchronous machines, in 2009 International Conference on Electrical Machines and Systems, 2009, pp. 16.

[11] S.-K. Sul, Control of electric machine drive system. 2011, p. 424.

[12] P Vas, Parameter estimation, condition monitoring, and diagnosis of electrical machines. New York: Oxford University Press, 1993. 